\title{
LAS POLÍTICAS DE SALUD DEL BANCO MUNDIAL
}

\section{THE WORLD BANK'S HEALTH POLICIES}

\author{
Shiri Noy*
}

\author{
RESUMEN
}

El Banco Mundial es un importante agente financiero externo en el área de la salud para los países en vías de desarrollo. Sin embargo, muchos estudios acerca de las reformas en el sector de la salud en estos países, no toman en cuenta el papel que las instituciones financieras internacionales pueden tener en estos casos. En este artículo, se analizan las políticas de salud que ha implementado el Banco Mundial desde 1975, analizando el rol que ha ejercido en la salud global y en su política de salud. Asimismo, se estudia la evidencia empírica sobre el efecto del Banco Mundial en el sector de la salud en los países en desarrollo, centrándose en las experiencias de países en América Latina.

PALABRAS CLAVE: BANCO MUNDIAL * AMÉRICA LATINA * POLÍTICA DE LA SALUD * AYUDA SANITARIA * DESARROLLO ECONÓMICO Y SOCIAL * PAÍSES EN DESARROLLO

\section{ABSTRACT}

The World Bank is an important external funder of health aid to developing countries. However, many accounts of health sector change and reform do not account for the role that international financial institutions may play in these policy changes. In this paper, I review the World Bank's health policies since 1975, discussing its changing role and policies for developing countries' health policies. I then summarize the existing empirical evidence for the effect of the World Bank in developing countries' health sectors, focusing on Latin American countries.

KEYWORDS: WORLD BANK $*$ LATIN AMERICA $*$ HEALTH POLICY $*$ HEALTH AID $*$ ECONOMIC AND SOCIAL DEVELOPMENT *DEVELOPING COUNTRIES

* Department of Sociology, University of Wyoming, Laramie, Wyoming, Estados Unidos. snoy@uwyo.edu 
El Banco Mundial es un actor importante en el campo de la salud a nivel global, debido a que es el agente de financiamiento externo más grande de este sector, gastando más de un billón de dólares cada año en proyectos de salud, nutrición y población en países en desarrollo (Ruger, 2005 y Fair, 2008). Desde finales de los años 70, el Banco Mundial ha invertido en proyectos de reforma en el sector de la salud; no obstante, varias personas han argumentado que su involucramiento ha dañado al sistema público de salud en muchos países en vías de desarrollo, debido al impulso de sus políticas neoliberales.

En este artículo se describe la evolución de las políticas que ha implementado el Banco Mundial en el campo de la salud, mediante el análisis de los documentos en donde se declaran estas políticas, datos y la examinación de la literatura secundaria sobre el rol del Banco Mundial en reformas de salud en países como Chile, Colombia y México.

\section{HISTORIA BREVE DEL BANCO MUNDIAL}

El Banco Mundial (BM) fue fundado junto con el Fondo Monetario Internacional (FMI) en la Conferencia de Bretton Woods realizada en New Hampshire, en el año de 1944, donde se incluyeron en total 44 naciones. El objetivo del Fondo Monetario Internacional fue promover la estabilidad económica internacional, ayudando a los países a manejar su economía; mientras la misión del Banco Mundial (entonces el Banco Internacional de Reconstrucción y Desarrollo) fue promover el desarrollo económico en países pobres y ayudar a otros países a reconstruir sus quebrantados sistemas económicos después de la Segunda Guerra Mundial (Babb, 2009: 41-42). Tradicionalmente, el Banco Mundial es encabezado por un ciudadano de origen estadounidense, mientras que el presidente del Fondo Monetario Internacional es de origen europeo. Los directores ejecutivos, representando los 179 países miembros del Banco Mundial (Driscoll, 1996: 4), constituyen la Junta Directiva y por lo general, se reúnen dos veces a la semana para supervisar el préstamo y la financiación de decisiones, así como, otros asuntos administrativos.
El Banco Mundial está compuesto por dos organizaciones principales: el Banco Internacional de Reconstrucción y Desarrollo (BIRD) y la Asociación de Desarrollo Internacional (IDA), fundada en 1960. El Banco Mundial tiene alrededor de 7000 empleados (aproximadamente, tres veces más que el Fondo Monetario Internacional) y mantiene alrededor de 40 oficinas a nivel global, aunque el $95 \%$ de su personal trabaja en su oficina central ubicada en Washington, DC (Driscoll, 1996: 3).

Entre más pobre sea un país, sus condiciones de préstamo son más favorables: los países en vías de desarrollo cuya renta nacional per cápita (GNI) excede $\$ 1195$, pueden tomar un préstamo del Banco Internacional de Reconstrucción y Fomento, mientras que IDA solamente presta a gobiernos de naciones en desarrollo muy pobres, cuya renta per cápita (GNI) se encuentra por debajo de $\$ 1195$, esto para el año 2012 (IDA, 2012). Los préstamos del BIRD llevan una tasa de interés ligeramente por encima de la tasa de mercado y debe ser reembolsado dentro de 12-15 años, en cambio los préstamos de IDA son sin interés y tienen un vencimiento de 35 a 40 años. En contraste, todas las naciones miembros, tanto ricas como pobres, tienen el derecho a la asistencia financiera del Fondo Monetario Internacional-FMI (Driscoll, 1996: 4). En prestación a países en vías de desarrollo, el Banco no compite con otras fuentes de finanzas. Este asiste solo a aquellos proyectos para los cuales el capital requerido no está disponible a través de otras fuentes en términos razonables (Driscoll, 1996: 6).

Durante los primeros años, los Estados Unidos controlaron el $37,2 \%$ de los votos, lo cual les permitió el control en los cambios del Convenio Constitutivo del Banco, aunque las decisiones de modificar préstamos individuales requirieran una mayoría de votos del $80 \%$ (Babb, 2009: 42). Por lo tanto, las instituciones financieras internacionales han sido criticadas por ser un vehículo para los intereses estadounidenses $y$ principalmente, de ser promovedoras de intereses de países ricos debido a que su estructura de votos y toma de decisiones están basadas en las contribuciones financieras que dan al Banco mismo. 
LAS POLÍTICAS DE SALUD DEL BANCO MUNDIAL

El Banco Mundial tiene una larga historia de inversión en el área de salud y sigue siendo un agente financiero externo importante en muchas regiones del mundo, como se demuestra en el gráfico 1.

GRÁFICO 1

DESEMBOLSOS AL SECTOR SALUD DEL BANCO MUNDIAL POR REGIONES GLOBALES 1990-2011

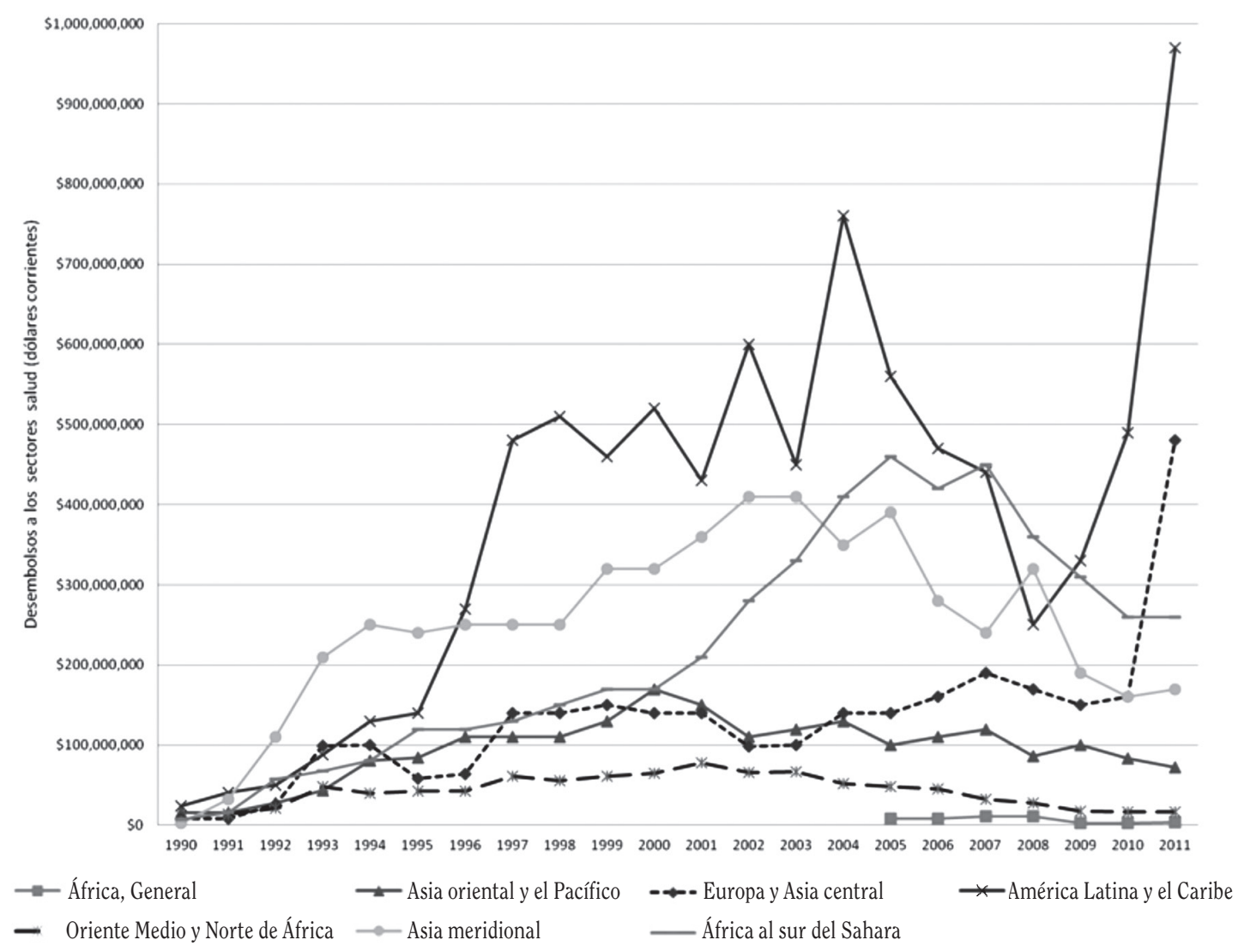

Fuente: Institute for Health Metrics and Evaluation (IHME), Development Assistance for Health Country and Regional Recipient Level Database 1990-2009.

Aunque los datos cuantitativos confiables existen solamente desde 1990, las políticas de salud del Banco Mundial tienen una historia mucha más larga. El primer documento del Banco Mundial que directamente habla de políticas de salud, es el Health Sector Policy Paper, el cual fue publicado en 1975 (Fair, 2008), asimismo, en este se vincula el desarrollo económico y el mejoramiento de las condiciones de salud (World Bank, 1975). En este reporte se discutió dos opciones para el involucramiento del Banco en el área de la salud en países prestatarios: la primera, era incorporar los beneficios al sector de la salud dentro de las actividades y préstamos existentes y la segunda, empezar a prestar a los servicios básicos en salud. En 1975, el Banco optó seguir con la primera opción (Fair, 2008).

Según Stout et ál. (1997), los obstáculos percibidos en la concesión de préstamos separados para los servicios básicos de salud, los cuales inicialmente disuadió el Banco, incluyó: la preocupación por la viabilidad de bajo costo de los sistemas sanitarios, la falta de voluntad política de los gobiernos para empezar reformas significativas y los asuntos relacionados con la 
función propia del Banco en el sector. No obstante, el Consejo de Administración del Banco aprobó el primer préstamo para el sector de la nutrición — de 19 millones de dólares-a Brasil, en 1976 (Fair, 2008).

Después de unos años, el Banco decidió seguir la segunda opción formalmente y creó en octubre de 1979, el Departamento de Población, Salud y Nutrición (Health, Nutrition and Population Department, HNP) (Ruger, 2005). En 1980, el Banco publicó un segundo Health Sector Policy Paper, el cual marcó la disposición del Banco para prestar directamente al sector de la salud (World Bank, 1980). En esta fase, el objetivo principal del Banco fue ampliar el acceso a la asistencia médica. La estrategia se centró en la necesidad de servicios básicos de salud, especialmente en zonas rurales y en proyectos para desarrollar infraestructura básica de salud, reforzar la logística y el suministro de medicamentos esenciales, así como proporcionar servicios de salud materna e infantil, mejorar la planificación familiar y el control de enfermedades (Fair, 2008).

El primer préstamo al sector de la salud fue aprobado a Tunisia en 1981. Esta etapa marcó una amplificación de los proyectos del Banco: este empezó con un enfoque en crecimiento económico del Producto Nacional Bruto, pero ahora su visión sobre el desarrollo evolucionó, incorporando también el desarrollo humano (Kapur et ál., 1997). Esta decisión del Banco fue influenciada por la importante adopción de la Declaración de Alma Ata en la Conferencia Internacional de Salud Primaria, en 1978. Esta declaración marca un importante cambio en la visión de la salud, al definirla como un derecho humano fundamental, una meta social importante para el mejoramiento económico (Bloom y Canning, 2003). Con la misma Declaración se define para los gobiernos, el objetivo de proveer atención a la salud para todos en el año 2000, lo cual popularizó el modelo de atención primaria a la salud.

En 1986, se publicó el tercer importante documento vinculado con las políticas de salud del вм: Financing health services in developing countries: an agenda for reform. Anteriormente, el enfoque estuvo ligado en proveer servicios de salud a los segmentos más pobres de la población, vinculando esta inversión en salud al progreso económico. Adicionalmente, fue justificado de acuerdo a una lógica económica: el mercado falló en la provisión y financiamiento de los servicios de salud y por esta razón, se justificó el involucramiento del gobierno en una distribución más equitativa de los servicios (Stout et ál., 1997). En contraste, en Financing health services in developing countries: an agenda for reform, el énfasis fue en mejorar la eficiencia $y$ la equidad en la salud: "el problema no es sólo - tampoco es principalmente-que los sistemas de salud de los países en vías de desarrollo están basados en los hospitales. Es que el gasto público en salud está orientado a los hospitales, y por lo tanto beneficia a los ricos desproporcionadamente" "(World Bank, 1986: 32).

En estos años, el BM también se centró en estudios sobre la financiación de los sistemas de salud, combinando el enfoque en atención primaria con un crecimiento económico lento $y$ déficits presupuestarios en muchos países. En este sentido, estaba interesado en la recuperación de costos y gastos públicos en el sector de la salud, a través de la implementación de tarifas de usuarios, algo que era y sigue siendo muy controversial (Gottret y Schieber, 2006).

El cuarto documento importante es el reporte de desarrollo mundial de 1993, un reporte que el BM publica cada año, pero que en este año se enfocó en la salud: The World Development Report 1993: Investing in Health. El informe analiza tres enfoques para orientar las políticas con el fin de mejorar el área de salud en los países en vías de desarrollo y en países anteriormente comunistas (Fair, 2008). En primer lugar, se reconoce la importancia de la toma de decisiones de salud a nivel de los hogares, argumentando que los gobiernos deben aplicar políticas de crecimiento económico que beneficien a la población pobre y que permitan mejorar la toma de decisiones, a través del empoderamiento económico. En segundo lugar, promovió la redirección del gasto estatal en salud hacia programas rentables, incluyendo la prevención, promoción y divulgación,

$\overline{1 \quad \text { Traducción propia. }}$ 
además de definir un paquete mínimo de servicios médicos esenciales que benefician prioritariamente a los pobres. Por último, se alentó una mayor diversidad y competencia en la prestación de financiamiento y servicios de salud.

Fair (2008) describe esta etapa, que comenzó en 1987, como una etapa de reformas de salud, un cambio grande de la etapa anterior de atención primaria a la salud (1980-1986). En muchos sentidos, era un cambio de modelo, de proveer más servicios y monitoreo de las condiciones de la salud hacia un involucramiento más directo en la organización de los mismos sectores en los países con quienes trabaja el Banco.

Desde 1997, con la publicación del quinto documento clave, el informe sectorial Health, nutrition, and population sector strategy paper, el enfoque consistió en afirmar lo que el вм puede aportar: conocimiento de los países en vías de desarrollo, su enfoque multi-sectorial $y$ macro a nivel de estos, y su capacidad de movilizar grandes recursos financieros directamente o a través de asociaciones con otras organizaciones (Fair, 2008). No era la primera vez que el Banco discutió la importancia de su involucramiento en los sectores de salud en los países en vía de desarrollo: "promover mejoras en las finanzas del sector de salud se encuentra entre las más valiosas contribuciones que puede hacer el Banco Mundial para mejorar la atención de salud en países de bajos ingresos"2 (World Bank, 1986: 63), pero en 1997, se aclaró las competencias específicas que el Banco pudo aportar en este tema.

En esta etapa, el enfoque fue en los cambios institucionales y en los logros de salud para la población pobre e indigente. En 2001, el Bм siguiendo con sus objetivos discutidos anteriormente, también apoyó al trabajo para lograr los Objetivos de Desarrollo del Milenio (Millennium Development Goals, MDGS). Los MDGs son 8 objetivos internacionales, que 193 estados miembro de las Naciones Unidas y 23 organizaciones internacionales han consentido lograr para el año 2015.

El sexto documento publicado en 2007 , es el más reciente e importante que delinea la estrategia de salud del BM, este es el Healthy

2 Traducción propia. development: the World Bank strategy for health, nutrition and population results. Este documento sigue con las líneas generales de los documentos y agendas anteriores del Banco, pero tiene más énfasis en mejorar los logros en salud de la población (que corresponde a los MDGs), principalmente, los logros de las personas de escasos recursos.

\section{EL BANCO MUNDIAL, NEOLIBERALISMO Y SALUD}

Muchos han escrito sobre el neoliberalismo, desde su encarnación en el formulario del Consenso de Washington por las instituciones financieras internacionales y más recientemente, el post-Consenso de Washington. Mientras que un tratamiento exhaustivo de la evolución de estas ideas y las diversas facetas del neoliberalismo están más allá del alcance de este artículo, se examinará brevemente los principios centrales del neoliberalismo y del Consenso de Washington.

El neoliberalismo ha sido clasificado de muchas formas, desde un enfoque económico a una ideología e incluso una filosofía. Sin embargo, el neoliberalismo se caracteriza por la idea de que los mercados son la forma más eficiente para la distribución de recursos, lo que caracteriza una economía sana. Subyacente a esta creencia, las ventajas de que el mercado asigne los recursos es el énfasis sobre la propiedad y los contratos, como medio de racionalizar la economía. La continuidad natural de esta idea, es que la economía es mejor dejarla al sector privado, en lugar del gobierno.

El Consenso de Washington fue acuñado en 1989, con la finalidad de describir el típico paquete de reformas promovidas por las instituciones financieras internacionales $y$ los Estados Unidos para los países en vías de desarrollo. Entre las recomendaciones estuvieron el comercio y la liberalización financiera, la privatización de empresas estatales y la protección jurídica de los derechos de propiedad; en particular interés se encuentra la discusión del gasto público. Según Williamson (2002), el economista que desarrolló el documento del que nace la lista de políticas conocidas como el Consenso de Washington, aboga por el gasto público, 
el cual es "pro-pobres y pro-crecimiento"; es decir, el gasto público se considera necesario $y$ apropiado, por ejemplo, si este es para salud básica, educación e infraestructura, en lugar de ser utilizado en defensa o administración. Naim (2000: 506) ha argumentado que nunca hubo un consenso y que los economistas estaban en desacuerdo, no solo sobre el contenido, sino también sobre el ritmo y la secuencia de las reformas, asimismo, señaló que "confusión de Washington" podría ser un mejor descriptor que "Consenso de Washington". Evidentemente, la realidad estaba en contradicción con las recomendaciones, por lo menos en lo que respecta a los gastos públicos. Williamson establece que: "el menor avance fue en la aplicación de la segunda directiva, reorientar las políticas de gasto público"3(1999) y el "Consenso de Washington se hizo lo que hizo, no de lo que se dijo [que hizo]"4 (Kanbur, 1999) y lo que hizo es achicar los sectores públicos sociales. No obstante, el Consenso de Washington original indica que al menos algunos economistas eran conscientes de que el gasto en salud y la educación son importantes, no solo por la equidad, sino porque podrían ayudar a reducir la pobreza, lo cual sería beneficioso para el crecimiento económico, debido a la conveniencia de trabajadores educados y sanos.

El término post-Consenso de Washington surgió a fines de la década de 1990 y refleja la necesidad de ir más allá del Consenso de Washington tras considerar seriamente la importancia de las instituciones nacionales en cualquier esfuerzo de reforma (Onis y Senses, 2005; Stiglitz, 1998 y 2003; Burki y Perry, 1998; Clift, 2003 y Radin, 2008). El post-Consenso de Washington representa una respuesta al "creciente papel de los parlamentos, los tribunales, $y$ las organizaciones no gubernamentales para resistir las iniciativas de reforma" ${ }^{5}$ (Naim, 2000: 506) y la petición de reforma con un rostro humano (Ilon, 1996). Además, algunos han argumentado que contrariamente al Consenso de Washington en el marco del post-Consenso,

\begin{tabular}{ll}
\hline 3 & Traducción propia. \\
4 & Traducción propia. \\
5 & Traducción propia.
\end{tabular}

las inversiones en capital humano han sido vistas por las Instituciones Financieras Internacionales (IFIs) como un fin en sí mismo y no solo por su contribución al crecimiento económico; por ejemplo, a partir de finales de los años 90, el BM comienza a considerar el Índice de Desarrollo Humano (una medida elaborada por las Naciones Unidas para el Desarrollo en 1990, la cual incluye estadísticas del estado nutricional, los logros educativos y estado de salud) como un indicador importante de la pobreza (Bonal, 2002). En comparación con el indicador anterior (PIB por habitante), esta medida incorpora claramente las dimensiones sociales del desarrollo; sin embargo, como su predecesora, el post-Consenso de Washington "sigue siendo imbuida de confusión teórica y zonas grises"6 (Santiso, 2004: 829).

Más ampliamente en los estudios de desarrollo, el post-Consenso de Washington estableció la primacía del Estado en lo que concierne al desarrollo. Anteriormente, se teorizó el proceso de desarrollo como el producto de factores externos: los precios determinados externamente, la ayuda externa, etc. El post-Consenso de Washington en reacción directa a las fallas del Consenso, estableció que los legisladores nacionales, mediante la aplicación de "buenas" políticas nacionales, podrían hacer la diferencia en la situación de la economía (Naim, 2000: 512). Algunos sostienen; sin embargo, que las conversaciones de desplazarse más allá del Consenso de Washington son exagerados por varias razones: la intervención del Estado sigue siendo desanimada, excepto en el caso de "las imperfecciones del mercado", el Estado sigue siendo visto como una entidad egoísta, impulsada por agentes de búsqueda de rentas, proclive a la corrupción (Fine, 2001 y Bonal, 2002).

Empíricamente, estudios que han examinado directamente los efectos de las presiones neoliberales sobre el gasto en salud en América Latina, han planteado diversas conclusiones. Hunter y Brown (2000) no encuentran una asociación entre préstamos del BM en un país determinado y los recursos dedicados a la educación y salud. Noy (2011) concluye que en América Latina, la presencia de instituciones

6 Traducción propia. 
financieras internacionales, incluido el Banco Mundial, no está asociada con menores niveles de gasto público en salud como el porcentaje del PIB. No obstante, como se describirá en la siguiente sección, han tenido impactos importantes sobre la estructura del sector salud (los servicios $y$ los seguros de salud).

\section{EL BANCO MUNDIAL Y LAS REFORMAS EN SECTORES DE LA SALUD EN LATINOAMÉRICA: UN BREVE RESUMEN}

Actualmente, existen diversas publicaciones del efecto del Banco Mundial sobre las reformas de los sectores de la salud en países latinoamericanos. La mayoría ofrecen investigaciones cualitativas del cambio (por ejemplo, De Vos et ál., 2005; Homedes y Ugalde, 2005; Barrientos y Lloyd-Sherlock, 2000; De Beyer et ál., 2000; Buse y Gwin, 1998) y critican severamente los cambios neoliberales que han promovido las instituciones financieras internacionales.

\section{CHILE}

Los dos casos más estudiados probable-mente son Chile y Colombia (Homedes y Ugalde, 2005 y 2010). El primero recibe mucha atención debido a que en el régimen de Pinochet, los Chicago Boys (economistas educados en la Universidad de Chicago, Estados Unidos) implementaron muchas reformas neoliberales $y$ entre ellas, reformas en los sectores de la salud. Se podría decir que Chile ha tenido las reformas neoliberales más radicales en el mundo. Específicamente, en los años 80, el gobierno creó las Instituciones de Salud Previsional (ISAPREs); es decir, seguros privados de salud (que pueden ser con fines de lucro) (Unger et ál., 2008; Ewig y Kay, 2011). Los empleados formales pueden comprar la cobertura de una ISAPRE por un costo determinado en el mercado ( $y$ por riesgo de salud, con mujeres pagando hasta $400 \%$ más en cuotas que los hombres) o pueden afiliarse con un seguro estatal. El Fondo Nacional de Salud (FONASA) es la institución pública de seguros y financiamiento de salud, el cual fue creado en 1979; la infraestructura de provisión de servicios fue después consolidada y renombrada como Sistema Nacional de Servicios de Salud (SNSS). Las personas sin capacidad de pago, que no podían adquirir un seguro de las ISAPREs, podían recibir servicios médicos de FONASA o de un médico privado subsidiado por FONASA, pero esta opción tenía también costos por parte del usuario (Homedes y Ugalde, 2005). En la década de 1980, bajo el Gobierno de Pinochet en Chile, la entrega de atención médica fue abierta al sector privado $y$ descentralizado a nivel municipal, lo cual en gran medida ha agravado las desigualdades existentes (Unger et ál., 2008).

\section{COLOMBIA}

Por otro lado, Colombia es famosa por su Ley 100 , cuyo objetivo fue un intento de mejorar el sistema impuesto en Chile. En este sistema, las personas pueden afiliarse al sistema de seguro de salud, el cual ofrece un paquete de servicios médicos estandarizado. Los afiliados asalariados contribuyen con el $12 \%$ de su sueldo $y$ el gobierno subsidia a los indigentes. El sector privado también puede participar a través de las Entidades Prestadoras de Salud (EPSS). Estas entidades establecen contratos con clínicas, hospitales públicos y privados, o también pueden operar sus mismos establecimientos de salud. Este sistema ha tenido bastantes problemas debido a la incapacidad del gobierno para pagar los servicios de los indigentes y los costos subsiguientes (Homedes y Ugalde, 2005).

\section{MÉXICO}

Las reformas en el sistema de la salud mexicano y específicamente, su descentralización iniciada en 1980, es una reforma que también impulsó el Banco Mundial: "en 1983, México aceptando la exigencia del Banco Mundial para obtener préstamos y renegociar la deuda externa descentralizó los servicios de salud de la Secretaría de Salud (SSA)" (Werld Bon K., 1986). Entre 1988-1994, el gobierno paralizó el proceso de descentralización (que fue implementado parcialmente, es decir, solo por unos estados mexicanos), porque "el gasto que ocasionó el proceso de descentralización fue enorme y los resultados decepcionantes" 
(Homedes y Ugalde, 2010: 86). En los años siguientes, la descentralización avanzó aún más, empezando con el gobierno que tomó poder en 2000-2006 y el cual nombró como ministro de salud al Dr. Julio Frenk, quien trabajó con el вм en las reformas del sistema de salud colombiano. En el año 2004, el gobierno mexicano inició el Seguro Popular (SP), un seguro voluntario para que las familias que no eran beneficiarias de la seguridad social, pudieran afiliarse. La SSA pagaba la póliza de las personas que estuvieran en la categoría del $20 \%$ de ingreso más bajo y conservaba el precio para otras familias con un ingreso estable (Homedes y Ugalde, 2010). Según estos mismos autores, las reformas han impactado al sistema negativamente, ya que se ha fragmentado y "la reforma casi ha doblado el gasto per cápita, no ha disminuido la ineficiencia, no ha mejorado la productividad ni la calidad, $y$ ha aumentado la inequidad entre los estados" (2010: 87).

El análisis de la participación y los efectos del вM sobre las reformas de los sectores de salud en estos tres países, son informativos, pero analiza los casos más extremos y por lo tanto, pueden exagerar la capacidad del вм de influir en los sectores de la salud en países en vías de desarrollo. Aunque Colombia y Chile son los casos más estudiados y tal vez los países con las reformas más profundas, las recientes investigaciones han dirigido su atención a otros países donde los efectos han sido menos profundos, pero donde el Bм ha desempeñado un gran papel en las reformas de los sectores de salud. Estos estudios, aunque en su mayoría no se centran en el papel del BM, sí han documentado los cambios y mecanismos de influencia de este, entre ellos se encuentran: Ewig (2006) sobre la reforma del sector de la salud y género en Perú; Lloyd-Sherlock (2005) sobre la reforma del sector de la salud en Argentina y, Homedes y Ugalde (2005), quienes han analizado la participación del BM en la reforma del sector de la salud en El Salvador.

Empíricamente, los estudios existentes no proporcionan evidencia directa acerca de si efectivamente los préstamos del Fondo Monetario Internacional y del Banco Mundial resultan en menor gasto público en el área de la salud, como fue discutido anteriormente; no obstante, se ha logrado documentar su efecto perjudicial sobre otros indicadores sociales: educación (Bonal, 2002; Ilon, 1996; Hunter y Brown, 2000), la pobreza (Easterly, 2000) y las pensiones (Mesa-Lago, 2006 y 2008; Mesa-Lago y Müller, 2002). Los estudios sobre la reforma del sector salud en América Latina han señalado que existe una mayor privatización y que los IFIs promueven el interés de las empresas extranjeras y agencias de seguros (Armada et ál., 2001; Barrientos y Lloyd-Sherlock, 2000; De Vos et ál., 2006; Homedes y Ugalde, 2005).

\section{CONCLUSIÓN}

Este artículo ha proporcionado un análisis sistemático de las políticas cambiantes del Banco Mundial en la salud global, usando documentos primarios propios de este $y$ estudios secundarios sobre el rol del mismo en reformas específicas de sectores de salud en países como Chile, Colombia y México, con datos cuantitativos descriptivos del gasto en salud del Banco según región mundial. El BM ha tenido y sigue teniendo un rol importante en los sectores de la salud de muchos países en vías de desarrollo. Este ha cambiado su modelo, pasando cinco grandes fases desde 1975, cuando formalmente empezó a involucrarse en los sistemas de salud con proyectos independientes (antes había componentes de salud, los cuales cual formaron parte de otros proyectos).

Como un actor importante en el campo de salud global -en su función financiera así como, en su función normativa- es relevante que investigadores, especialmente de sistemas de salud en países en desarrollo, consideren el impacto de la globalización y los actores internacionales en estas reformas. El BM ha sido acusado de promover una agenda de privatización, favoreciendo los intereses de corporaciones transnacionales - principalmente de seguros de salud $y$ de empresas farmacéuticas - pero los estudios cuantitativos han sido pocos y los estudios cualitativos se enfocan en los casos más extremos en Latinoamérica: Chile y Colombia. Este artículo ha argumentado que el Banco Mundial ha cambiado su orientación y sus propias políticas de salud. Es necesario examinar 
empíricamente si estos cambios declarados han sido implementados en las políticas (si este cambio en planeamiento se ha manifestado en las políticas actuales) en sus proyectos, su financiamiento $y$ sus consejos a gobiernos en el campo de salud. También es importante investigar como diferentes países han recibido, negociado o resistido la influencia del Banco Mundial.

Sus métodos y la filosofía del neoliberalismo han sido controversiales en este caso, asimismo, en muchas maneras, el BM ha cambiado su método $y$ ha reconocido la importancia de las instituciones $y$ condiciones locales en la implementación y planificación de sus proyectos. Es importante que quienes estudian la salud pública y sus reformas, consideren el impacto de las instituciones financieras internacionales, entre ellas, el Banco Mundial, el cual ha tenido una gran influencia en los sistemas de salud de muchos países, especialmente en Latinoamérica. Con el fin de considerar este impacto, especialmente con una visión histórica, se debe entender las políticas cambiantes del вм en este sector, asimismo, es relevante analizar su efectividad y cómo se han reflejado en las políticas nacionales de países en desarrollo.

\section{REFERENCIAS BIBLIOGRÁFICAS}

Armada, Francisco; Muntaner, Carles y Navarro, Vicente. "Health and social security reforms in Latin America: the convergence of the World Health Organization, the World Bank and transnational corporations". International Journal of Health Services 31 (4). 2001: 729-768.

Babb, Sarah. "The social consequences of structural adjustment: recent evidence and current debates". Annual Review of Sociology 31. 2005: 199-222.

Babb, Sarah. Behind the development banks: Washington politics, world poverty, and the wealth of nations. Chicago, IL: University of Chicago Press, 2009.

Barrientos, Armando y Lloyd-Sherlock, Peter. "Reforming health insurance in Argentina and Chile". Health Policy and Planning 15 (4). 2000: 417-423.
Bloom, David y Canning, David. "The health and poverty of nations: from theory to practice". Journal of Human Development 7. 2003: 47-71.

Bonal, Xavier. "Plus ca change... the World Bank global education policy and the post-Washington Consensus". International Studies in Sociology of Education 1 (12). 2002: 3-22.

Burki, Shahid Javed y Perry, Guillermo E. Beyond the Washington Consensus: institutions matter. Washington DC: World Bank, 1998.

Buse, Kent y Gwin, Catherine. "The World Bank and Global Cooperation in health: the case of Bangladesh". Lancet 351. 1998: 665-669.

Clift, Jeremy. "Beyond the Washington Consensus". Finance \& Development. September 2003. En: <http://www.imf. org/external/pubs/ft/fandd/2003/09/pdf/ clift.pdf $>$ [Consultado el $1^{\circ}$ de junio de 2012].

De Beyer, Joy A.; Preker, Alexandar S. y Feachem, Richard G. A. "The role of the World Bank in international health: renewed commitment and partnership". Social Science \& Medicine 50. 2000: 169-176.

De Vos, Pol; De Ceukelaire, Wim y Van der Stuyft, Patrick. "Colombia and Cuba, contrasting models in Latin American Health Sector Reform”. Tropical Medicine and International Health 11 (10). 2006: 1604-1612.

Driscoll, David D. "The IMF and the World Bank: how do they differ?". 1996. En: <http:// www.imf.org/external/pubs/ft/exrp/differ/ differ.htm> [Consultado el 5 de junio de 2012].

Easterly, William. "The effect of IMF and World Bank programs on poverty". SSRN Working Paper Series. 2000. En: $<$ http://dx.doi.org/10.2139/ssrn.256883> [Consultado el 5 de junio de 2012].

Ewig, Christina. "Global Processes, Local Consequences: Gender Equity and Health Sector Reform in Peru". Social Politics: International Studies in Gender, State \& Society 13 (3). 2006: 427-455 
Ewig, Christina y Kay, Stephen J. "Retrenchment politics: policy feedback in Chile's health and pension reforms". Latin American Politics and Society 53 (4). 2011: 67-99.

Fair, Mollie. "From population lending to HNP results: the evolution of the World Bank's strategies in health, nutrition and population". IEG Working Paper 2008/3. 2008.

Fine, Ben. Social capital versus social theory. London: Routledge, 2001.

Gottret, Pablo y Schieber, George. Health financing revisited: a practitioner's guide. Washington, DC: World Bank. En: <http://siteresources.worldbank. org/InTHSD/Resources/ topics/HealthFinancing/HFrFull.pdf> [Consultado el 25 de mayo de 2012].

Homedes, Nuria y Ugalde, Antonio. "Neoliberalismo y salud. El engaño del Banco Mundial y FmI". Viento Sur 109. 2010: 83-87. En: <http://www. vientosur.info/articulosabiertos /vs109_ Neolibysalud_HemedesyUgalde.pdf > [Consultado el 15 de diciembre de 2013].

Homedes, Nuria y Ugalde, Antonio. "Why neoliberal health reforms have failed in Latin American". Health Policy 71. 2005: 83-96.

Hunter, Wendy y Brown, David. "World Bank directives, domestic interests, and the politics of human capital investment in Latin America". Comparative Political Studies 33. 2000: 113-143.

IDA. IDA Borrowing Countries. 2012. En: <http:// www.worldbank.org/ida/borrowingcountries.html> [Consultado el 19 de mayo de 2012].

Ilon, Lynn. "The changing role of the World Bank: education policy as global welfare". Policy and Politics 24 (4). 1996: 413-424.

Kanbur, Ravi. The strange case of the Washington consensus: a brief note on John Williamson's 'What should the bank think about the Washington Consensus?'. 1999. En: <http://www. people.cornell.edu/pages/sk145/papers/ Washington $\% 20$ Consensus.pdf $>$ [Consultado el 21 de mayo de 2012].
Kanbur, Ravi. "The development of development thinking". Journal of Social and Economic Development 6 (2). 2004: 147-158.

Kapur, Devesh; Lewis, John y Webb, Richard. The World Bank: it's first half century. Washington, DC: The Brookings Institution. 1997. En: <http:// siteresources.worldbank.org/ EXTARCHIVES/ Resources/wb_first_half_century.pdf> [Consultado el 21 de mayo de 2012].

Lloyd-Sherlock, Peter. "Health sector reform in Argentina: A cautionary tale". Social Science \& Medicine 60. 2005: 1893-1903.

Mesa-Lago, Carmelo. "Private and public pension systems compared: an evaluation of the Latin American experience". Review of Political Economy 18 (3). 2006: 317-334.

Mesa-Lago, Carmelo. Reassembling social security: a survey of pensions and health care reforms in Latin America. Oxford: Oxford University Press, 2008.

Mesa-Lago, Carmelo y Müller, Katharina. "The politics of pension reform in Latin America". Journal of Latin American studies 34. 2002: 687-715.

Naim, Moses. "Fads and fashion in economic reform: Washington Consensus or Washington confusion?". Third World Quarterly 21 (3). 2000: 505-528.

Noy, Shiri. "New contexts, different patterns? A comparative analysis of social spending and government health expenditure in Latin America and the OECD". International Journal of Comparative Sociology 52 (3). 2011: 215-244.

Onis, Ziya y Senses, Fikret. "Rethinking the emerging post-Washington Consensus". Development and change 36 (2). 2005: 263-290.

Radin, Dagmar. "World Bank funding and health care sector performance in Central and Eastern Europe". International Political Science Review 29 (3). 2008: 325-347.

Ruger, Jennifer Prah. "The changing role of the World Bank in global health". American Journal of Public Health 95 (1). 2005: 60-70. 
Santiso, Carlos. "The contentious Washington Consensus: reforming the reforms in emerging markets". Review of International Political Economy 11 (4). 2004: 828-844.

Stiglitz, Joseph. More instruments and broader goals: moving toward the postWashington Consensus. 1998 WIDER Annual Lecture. Helsinki, Finland. January 7, 1998. En: <http://www.wider. unu.edu/publications/annual-lectures/ en_GB/AL2/_files/7809186053429923 9/default/annual-lecture-1998.pdf> [Consultado el 12 de mayo de 2012].

Stiglitz, Joseph. "Democratizing the International Monetary Fund and the World Bank: governance and accountability". Governance 16 (1). 2003 : 111-40.

Stocker, Karen; Wiatzkin, Howard y Iriart, Celia. "The exportation of managed care to Latin America". The New England Journal of Medicine 340 (14). 1999: 11311138.

Stout, Susan; Evans, Alison; Nassim, Janet y Raney, Laura. "Evaluating health projects: lessons from the literature". World Bank Discussion Paper 356. Washington DC: World Bank, 1997.

Williamson, John. "What Washington means by policy reform". Latin American adjustment: how much has happened? John Williamson (ed.). Washington: Institute for International Economics, 1990.
Williamson, John. What should the bank think about the Washington Consensus?. 1999. En: <http://www. iie.com/publications/papers/paper. cfm?ResearchID $=351>$ [Consultado el 20 de mayo de 2012].

Williamson, John. Did the Washington Consensus fail?. 2002. En: <http://www. petersoninstitute.org/publications/ papers/paper.cfm?ResearchID $=488>$ [Consultado el 20 de mayo de 2012].

World Bank. Health sector policy paper. Washington, DC: World Bank, 1975.

World Bank. Health sector policy paper. Washington, DC: World Bank, 1980.

World Bank. Financing health services in developing countries: an agenda for reform. Washington, Dc: World Bank, 1986.

World Bank. World development report: investing in health. New York: Oxford University Press, 1993.

World Bank. Health, nutrition and population sector strategy. Human Development Network. Washington, DC: World Bank, 1997.

World Bank. "Healthy development: the World Bank strategy for health, nutrition and population results". Human development network. World Bank, April 24, 2007.

Fecha de ingreso: 02/07/2012 Fecha de aprobación: 17/05/2013 
